\section{Oxidising Agents and Vat-dyed Cotton}

IN the course of an investigation into the action of oxidising agents upon cotton dyed with vat dyestuffs, we have obtained evidence of a simple relationship between the stable potential set up when a platinum electrode is dipped into a dilute solution of sodium hypochlorite, and the amount of chemical modification produced when this solution acts, under standard conditions, upon pure cotton cellulose dyed with the reduced or leuco form of certain vat dyestuffs. These results may prove of interest to those engaged in a study of the chemistry of cellulose and similar poly. molecular compounds and also to those who are working on phenomena of oxygen transfer facilitated by a chemically labile substance.

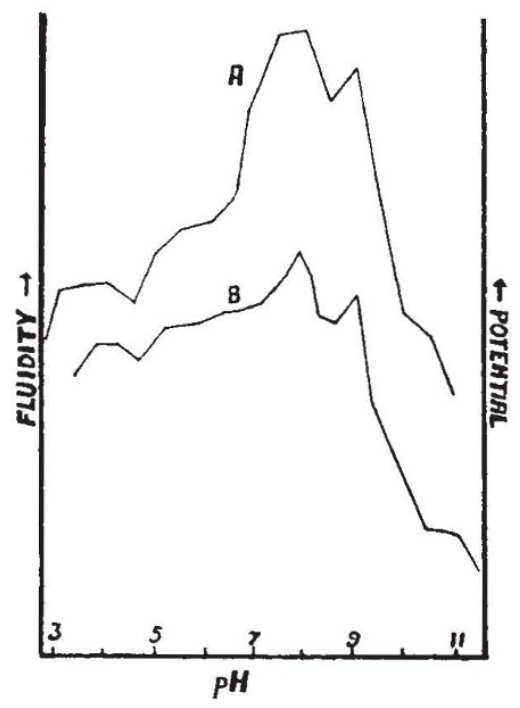

FIG. 1.

Certain vat dyestuffs exhibit great resistance to the action of chemical reagents in dilute solution, except that they are readily reduced at the essential quinone groups. The reduction- or leuco-compound is usually unstable, reverting easily to the vat dyestuff on contact with atmospheric oxygen or upon treatment with dilute aqueous solutions of oxidising agents. The presence on the cotton fibre of this reduced form of some vat dyestuffs causes a remarkable acceleration in the rate of chemical modification of the cellulose by dilute sodium hypochlorite. If this modification is measured by the increase in the fluidity (reciprocal of viscosity) of standard solutions of the treated cellulose in solutions of cuprammonium hydroxide, it can be shown that more modification is accomplished in a few seconds in the presence of reduced dyestuff than is brought about over a period of some hours by hypochlorite acting in the presence of the fully oxidised form of the dyestuff, or in the complete absence of vat dyestuff. In all these experiments light is excluded.

In a series of experiments, cotton dyed with a reduced vat dyestuff was treated, in the dark, for 10 minutes with dilute sodium hypochlorite solutions, maintained at a number of different hydrogen ion concentrations by a procedure in which the sodium hypochlorite is employed as its own buffering electrolyte. The connexion between the chemical modification of the cellulose (as measured by its fluidity) and the $p \mathrm{H}$ of the hypochlorite solution is given in curve $A$ on Fig. 1. In curve $B$ is shown the connexion between $p \mathrm{H}$ of the hypochlorite solution and the potential set up by it at a clean platinum electrode. This potential was measured in the usual way by forming a cell with the platinum electrode and a saturated calomel half element and measuring the E.M.F. of the cell potentiometrically. The potential at the platinum electrode is expressed by comparison with the hydrogen electrode in a solution of the same $p \mathrm{H}$ as the solution under examination.

The remarkable agreement between these two curves is not found in the unaccelerated oxidation of cellulose in the absence of reduced vat dyestuff. The agreement appears to indicate $(a)$ simple relationship between the cuprammonium fluidity and the mean molecular size of cellulose modified by accelerated hypochlorite oxidation, (b) a consistent and uncomplicated mechanism for the oxidation of cellulose by hypochlorite over a considerable range of hydrogen ion concentration, when the oxidation is accelerated by reduced vat dyestuff.

The use of reduced vat dyestuffs or analogous accelerating substances may lead to a simplification of the study of the oxidation of cellulose and similar compounds.

A fuller account of this work will appear in a forthcoming issue of the Journal of the Society of Dyers and Colourists.

\section{H. A. TURner. \\ G. M. NABAR.}

F. Scholefield.

Department of Textile Chemistry, College of Technology, Manchester 1. Nov. 7.

\section{Vision in the Ultra-Violet}

IN a letter in Nature of November 10 (p. 736) Prof. Fabry mentions two publications by Saidman on this subject, hitherto unknown to me, which I

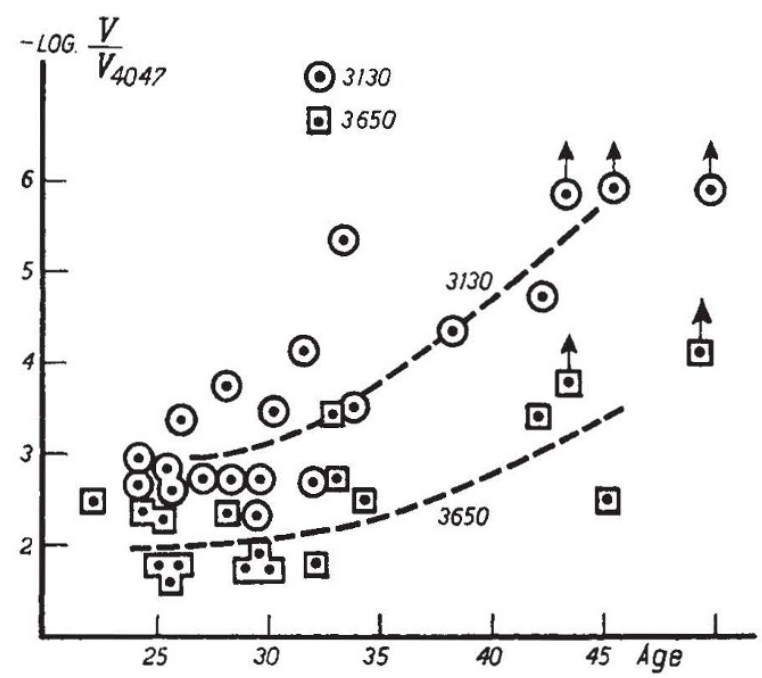

FIG. 1. Visibility for $\lambda \lambda 3130$ and 3650 relative to $\lambda 4047$ plotted against age. The points marked by arrows mean that the value of - $\log \left(V / V_{4047}\right)$ is certainly larger than indicated.

read with great interest. In one of them the correlation of the property of seeing ultra-violet light with 